\title{
Detection of GNSS Interference in Safety Critical Railway Applications using Commercial Receivers
}

\author{
Alessia Vennarini, Andrea Coluccia, Radiolabs \\ Daniel Gerbeth, Omar Garcia Crespillo, German Aerospace Center (DLR) \\ Alessandro Neri, Roma Tre University
}

\section{BIOGRAPHIES}

\begin{abstract}
Alessia Vennarini received the Master's degree in Information and Communication Technology Engineering from Roma Tre University in May 2011. She joined Radiolabs in April 2012 and she is involved in European (ESA and GSA) and national projects in activities related to characterization and analysis of GNSS signals.
\end{abstract}

Andrea Coluccia received the Master of Science in Advanced Communications and Navigation Satellite System at the University of Rome 2 "Tor Vergata". He joined RadioLabs in 2012 and he has been involved in GNSS European (ESA and GSA) and national projects. The main research topics are techniques for integrity monitoring, techniques for the interference detection and mitigation, design of local area augmentation networks for RAIL applications.

Daniel Gerbeth received a Bachelor and Master's degree in Electrical Engineering and Information Technology from Karlsruhe Institute of Technology in 2014. After working in the field of sensor fusion he joined the German Aerospace Center (DLR) in 2015. With the Navigation department he is involved in the research on future GBAS and integrity for navigation in the drone context.

Omar Garcia Crespillo holds a M.Sc. in Telecommunication Engineering from the University of Malaga, Spain. Since 2013, he is with the Navigation department of the German Aerospace Center (DLR). His current fields of research include resilient GNSS, inertial navigation, robust multi-sensor fusion algorithms and integrity monitoring for safe ground and air transportation systems.

Alessandro Neri is Full Professor in Telecommunications at the Engineering Department of the ROMA TRE University. Since December 2008, Prof. Neri is the President of the RadioLabs Consortium, a non-profit Research Center created in 2001 to promote tight cooperation on applied research programs between universities and industries. He received the Doctoral Degree in Electronic Engineering from "Sapienza" University of Rome in 1977. In November 1992 he joined the Electronic Engineering Department of Roma Tre University as Associate Professor in Electrical Communications and became Full Professor in Telecommunications in September 2001. His research activity is mainly focused on information theory, signal theory, signal and image processing, location and navigation technologies, and their applications to both telecommunications systems and remote sensing.

\begin{abstract}
Satellite navigation plays a critical role in new railway signaling and train control systems. It will allow higher levels of automation at a reduce cost in installation and maintenance as compared to current infrastructure-based technologies. However, in order to be integrated in railway systems, GNSS must guarantee the stringent railway safety requirements. GNSS safety has been extensively quantified for the aviation community, however, in the railway environment the probability of local GNSS hazards like interferences is larger due to its operation in a non-restricted space. Railway GNSS receivers must be therefore equipped with mechanisms to protect the position determination from being corrupted by undesired interferences (jamming or spoofing) without being aware of it. In this paper and based on Commercial-Off-The-Shelf (COTS) hardware we implemented interference detection functions suitable for railway localization. We detail the algorithm design as well as the necessary calibration procedure to determine the nominal signal model. The performance of the interference detection is analyzed with an experimental setup on a commercial train during the measurement campaign organized for the EU project ERSAT-GGC. Figures about the detection capability and detected events along the selected line are finally provided.
\end{abstract}




\section{INTRODUCTION}

In the last years, safety-critical applications are gaining attention by the scientific community and the receiver manufacturers. The goal is to introduce COTS receivers without the high costs typically associated with these applications. The peculiarity of safetycritical applications is the need to ensure the required performance in terms of integrity, continuity, and availability. The guidance of an aircraft during landing, the control of trains on high-density lines or the guidance of the docking of a cargo ship in specific situation are examples of such safety-critical applications. The work presented in this article is focused on the railway sector, to take advantage of real data acquired during several hours of a dedicated measurement campaign. Nevertheless, the methodology is applicable to other safety-critical applications.

The introduction of Global Navigation Satellite System (GNSS) positioning technology into the European Railway Traffic Management System (ERTMS) and in particular into the European Train Control System (ETCS) evolution roadmap is a key factor that can provide cost saving solutions for railway traffic management of local, regional and freight lines while guaranteeing interoperability.

As already happening in other application contexts, like the precision agriculture, geodesy or aeronautical positioning (the latter much similar to railway for the safety issue), the augmentation networks are used for providing corrections that improve GNSS positioning performance (e.g. accuracy and precision [1]) and barriers to protect the navigation system against hazard causes originating from the total Global Navigation Satellite System (i.e. the combined space \& ground segments and the on-board subsystems) and propagation effects. Examples of internal GNSS faults that can lead to system hazard causes are GNSS satellite fault, SBAS satellite fault, reference station fault. Specifically, for the railway sector, the use of a public and standard augmentation network has been confirmed in several projects ([2], [3], [4], [5]) as mandatory to support interoperable ERTMS safe train positioning.

While global or local augmentation can largely increase integrity and accuracy of GNSS positioning, the GNSS threats addressed with an augmentation network are not the only ones affecting the GNSS Signal-In-Space (SIS) on land. The presence of local GNSS threats such as multipath, Non-Line-Of-Sight (NLOS) and intentional/unintentional interference that are not detectable by a local or wide area augmentation network could corrupt the pseudorange measurements and lead to unbounded errors in the position estimation. One of the goals of the EU project ERSAT-GGC was to perform a classification of the railway tracks according to the expected presence of local GNSS hazards [6]. In this context in [9], we provided a preliminary survey of detection measures for each of the possible local hazard causes along the railway tracks. Also, different techniques have been implemented for the detection of multipath [7] and NLOS [8] for land-based applications. Finally, and in the main scope of this paper Radio Frequency Interference (RFI) represents one of the most dangerous threat for GNSS receivers.

The term RFI includes different sources of interference. There are intentional sources, like spoofers or jammers that specifically target the GNSS bands. But the interference can also come from unintentional sources like TV antennas, harmonic emissions from commercial high-power transmitters, ultra-wideband radar, or other in-band and near-out-of-band spill-over. GNSS receivers are vulnerable to both unintentional and intentional interferences as they can result in degraded navigation accuracy or in a complete loss of signal tracking. In this work we address the issue of detecting unintentional in-band and near-band interference using commercially available GNSS receivers for the railway application.

The use of commercial GNSS receivers based on COTS can offer a cost-effective solution to design algorithms for interference identification. In fact, some commercial receivers complement raw data information and GNSS data at the PVT level with additional receiver information including pre-correlation data such as Automatic Gain Control (AGC) and Power Spectral Density (PSD) measurements in terms of different GNSS frequencies and GNSS constellations.

A combination of different metrics has been considered in this work to reduce erroneous interference detection caused by other interfering signals. Particularly, a method for the identification of anomalies in the expected behavior of the AGC values as output of the Automatic Gain Control circuit opportunely combined with a GNSS signal spectrum monitoring technique is proposed. Both are pre-despreading techniques that evaluate the variation of the receiver signal power to assess the presence of interfering signals. AGC level monitoring aims at the detection of additional signals in the receiver frequency band that are normally not present. In fact, when the GNSS band is interference free, the AGC level is expected to be quite stable and confined within a known interval as the power level should be slowly varying [12]. Conversely, in case of an unlikely presence of interference, the AGC level drops sharply in response to increased power in the GNSS band [14]. In this sense, the variation of the AGC level can be used as a metric to detect the presence of interference.

The second method here considered is based on spectral monitoring, which works in the frequency-domain. Particularly, the starting assumption of this technique is the a-priori knowledge of the GNSS SIS spectrum at the antenna of the receiver. For example, for GPS satellites the nominal power level at the receiver is typically -157 dBW for the L1C channel. This level is much lower than the noise floor. Therefore, in nominal conditions, the observed spectrum should be similar to the one expected for white noise. These 
considerations are assumed valid also for the other GPS carrier frequencies like L2 and L5 and for other channels or constellations like GALILEO. An important step to properly apply this technique consists of the estimation of the spectrum. This impacts the capacity of the technique to correctly detect interfering signals characterized by a power level bigger than the noise power level.

In this work we present first a methodology to adapt the use of AGC and Power Spectral Density (PSD) information as interference detection techniques to the railway domain. The nominal values of AGC and PSD are highly dependent on the antenna and system installation on the train and therefore the derivation of thresholds for the detection process must be determined beforehand through a dedicated calibration process. The behavior of the detection metrics as well as the combined one, is analyzed with measurement data sets collected over different railway runs in the line Cagliari-San Gavino in Italy. In particular, the detection metrics are compared for two receivers that were installed on a commercial train: Septentrio AsteRx and Javad Delta. Finally, an assessment of the interferences detected during the measurement campaign is conducted to provide further insight about the expected presence of this threat in the railway domain.

The results suggest that the adaptation and combination of AGC and PSD metrics coming from COTS receivers for interference detection in the railway domain can be a cost-effective and receiver independent solution.

\section{STATISTICAL CHARACTERIZATION OF THE AGC AND PSD DETECTION METRICS}

In this work we utilize two different metrics to combinedly detect the presence of interference, namely the Automatic Gain Control (AGC) level monitoring and Power Spectral Density analysis.

The AGC is a hardware component of most receiver front-ends aiming at adapting the receiver input gain to keep quantization losses as low as possible. As shown in Figure 1, it is normally located between the analog portion of the front-end and the Analog-to-Digital Converter (ADC) to adjust the gain of the front-end to that of ADC input range [10].

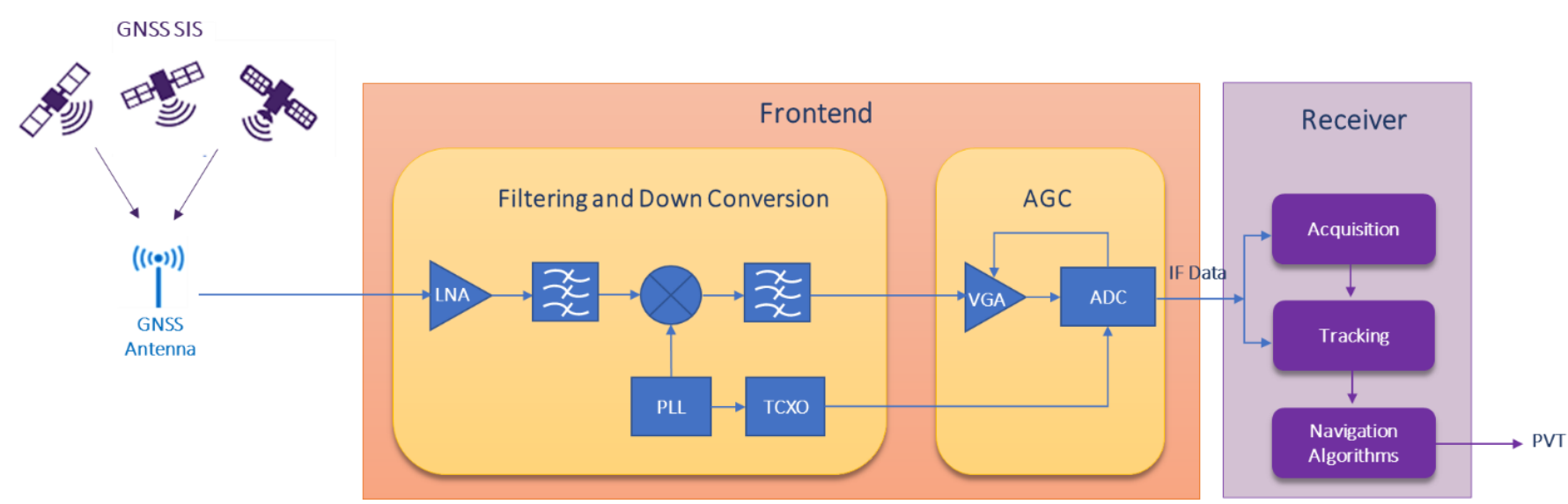

Figure 1: Typical GPS receiver architecture

In a GNSS receiver, the received analog signal is amplified, down-converted, filtered and then converted to a digital signal so that it can be processed by the acquisition and tracking loops. Quantization losses can occur during signal sampling and quantization and they depend on the ratio between the ADC's maximum quantization threshold, L, the number of bits utilized, and the incoming signal standard deviation, $\sigma[11]$. The AGC optimizes the $\mathrm{L} / \sigma$ ratio adapting the power of the incoming signal to minimize the digitalization losses. This functionality is implemented in any GNSS receiver that uses multibit quantization [12], [13].

When the GNSS band is interference free, the AGC level exclusively depends on the ambient noise rather than the signal power as the received signal power level is below of the thermal noise floor [13]. Therefore, in nominal condition, it is expected to be quite stable and limited within a known interval as the power level should be slowly varying [12]. Figure 2 shows a real example of AGC level trend acquired by the Septentrio AsteRx4 OEM receiver during a run of the ERSAT-GGC measurement campaign. In case of an unlikely presence of interference, the AGC level drops sharply in response to increased power in the GNSS band. In this sense, the variation of the AGC level can be used as a metric to detect the presence of interference [14]. The assumption is that interfering signals are more powerful than the authentic ones; therefore, the monitoring of receiver power measurements via the observation of the AGC level variation can provide an evidence of the presence of RFI [15]. 


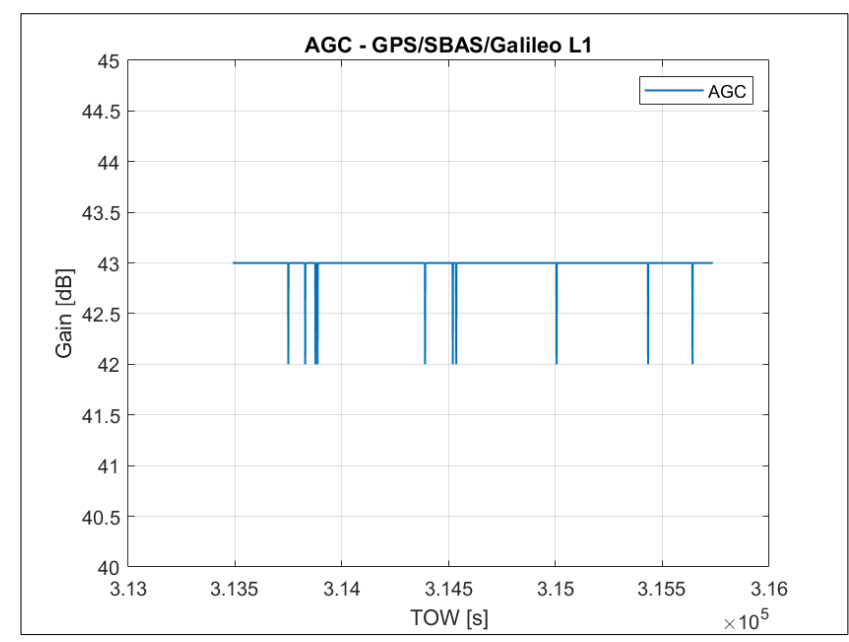

Figure 2: AGC level trend over time

A second, independent approach to detect interference in commercial GNSS is the monitoring of the power spectral density. It works in the frequency-domain via wave analysis methods applied on the GNSS SIS at the antenna of the receiver.

In nominal condition, the power level of the GNSS SIS, captured at the antenna level, is below the thermal noise that it is assumed to be white over the whole digitization bandwidth. In free-interference condition, the spectral estimate of the signal just after the receiver front-end is given by the equivalent transfer function of the front-end, multiplied by the variance of the noise that passed through the analog front-end [12]. Figure 3 shows a real example of time trend of the power spectral density of the signal in output to the AGC block of the Septentrio AsteRx4 OEM receiver. This PSD has been computed on data acquired during a run of the ERSAT-GGC measurement campaign.

Therefore, an interfering signal characterized by a power level bigger than the noise power level could be detected using properly the spectral estimate of the signal in output of the receiver frond-end. In particular, this spectral estimate is compared with a spectral mask defined in nominal free-interference condition.

The spectral estimate is the computation of the Power Spectral Density (PSD) of the GNSS received signal. Several methods can be used for this purpose, such as normalized Fast Fourier Transform (FFT) or periodogram methods.

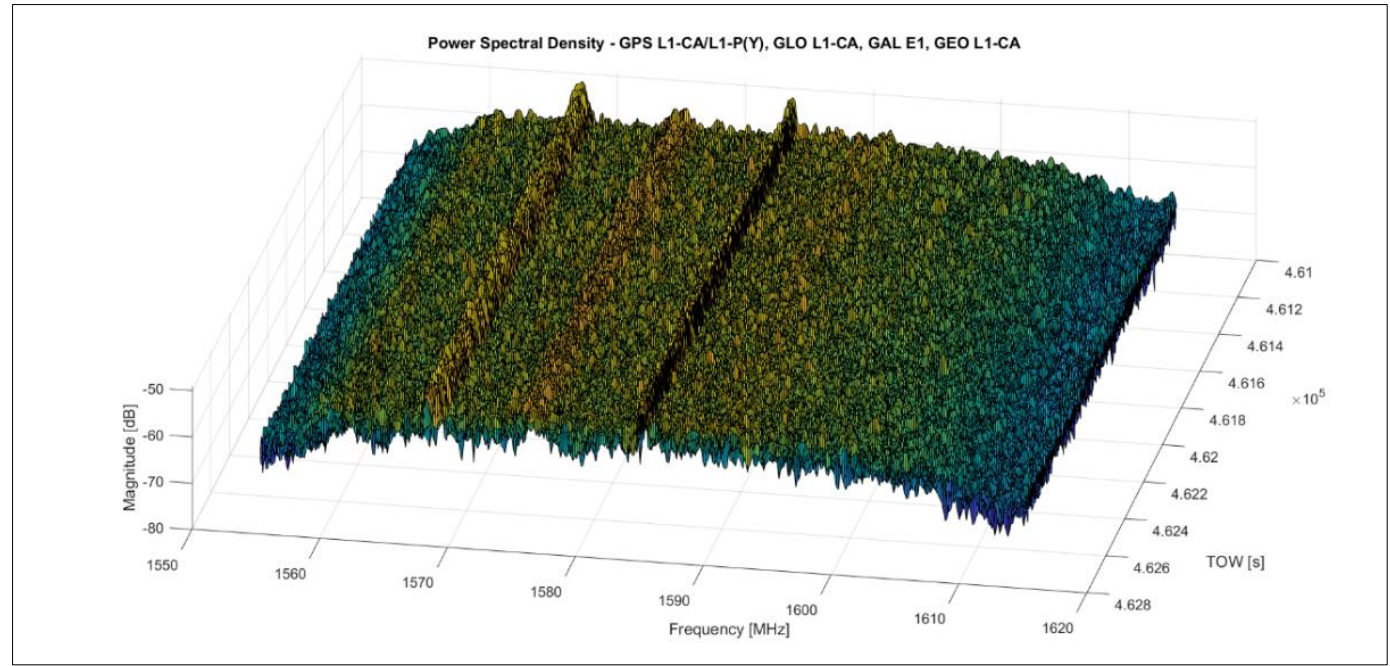

Figure 3: PSD trend over the course of 25 minutes

\section{THRESHOLD SETTING/DEFINITION}

In general, interference detection techniques try to detect if any distortion from the nominal conditions has been occurred in order to raise an alert and eventually carry out some possible mitigation strategy. Interfering signal with a power level exceeding the noise 
floor can be detected with the AGC and spectral analysis by comparing the PSD of the received signal and the level of AGC with a decision threshold.

The received GNSS signal power is always below that of the thermal noise floor power, $P_{n}$, that it is given by the following equation:

$$
P_{n}=k T_{A} B W
$$

where $k$ is the Boltzmann's constant, $T_{A}$ is the effective antenna temperature, and $B W$ is the bandwidth [10]. If it is also considered the additive noise from the first stage front end components (see Equation (2), where $T_{R}$ is the receiver noise temperature derived from Friis formula and the cascade of the front-end components), this means that the noise is the dominant source in the raw intermediate frequency GNSS data samples [10].

$$
P_{\text {ntotal }}=k\left(T_{A}+T_{R}\right) B W
$$

The thermal noise floor is not constant, but it depends on the temperature. Small variations of the average received power can be expected as a function of the temperature and environmental changes around the antenna [12]. The dependence on the ambient temperature and on hardware components is the reason why algorithms based on PSD and AGC analysis require a calibration.

The calibration step is needed to adapt these detection techniques to the performance of every unique installation of the GNSS antenna on a train. As every antenna together with the structural parts around can change the receptions of GNSS signals, the sensitivity of the detections has to be calibrated for every measurement setup. This is necessary to account for the nominal noise and multipath levels of each installation in order to adjust the techniques to achieve the desired false alarm probabilities.

For this calibration no thermometer is required, as real-world temperature variation of the order of 50 degrees Celsius introduces only a small variation in in Kelvin scale.

In real operational scenarios the AGC compensates gain variations of the incoming signal passing through the Low Noise Amplifier (LNA) embedded in the active antenna and the front-end HW components, which noise figure depends on the receiving bandwidth and the ambient temperature, expressed in Kelvin scale, where the hardware is installed (see Equation (1) and (2)). In fact, when the ambient temperature increases, the LNA efficiency decreases and introduces a reduced gain, which is compensated by the AGC. Consequently, the interference detection threshold over AGC level monitoring has to be defined considering the averaged value of the AGC level, measured in nominal conditions [16]. As proposed in [16], a detection threshold, using AGC level values, can be derived as described in the following:

$$
t h r=\lfloor M-n \sigma\rfloor
$$

where $M$ and $\sigma$ are respectively the mean value and the standard deviation of the AGC levels measured in nominal conditions and $n$ is an integer number. Figure 5 shows a real example of AGC level threshold computed applying the threshold algorithm described above on data acquired during the ERSAT-GGC measurement campaign. In this example, the threshold has been computed using AGC level values depicted in Figure 2. Data acquired "in nominal condition" means that the received GNSS signal is not affected by RFI coming from the environment outside the train. From the AGC level point of view, data acquired in nominal condition comprise the incoming GNSS signal plus the eventually RF interference generated by any equipment installed onboard the train.

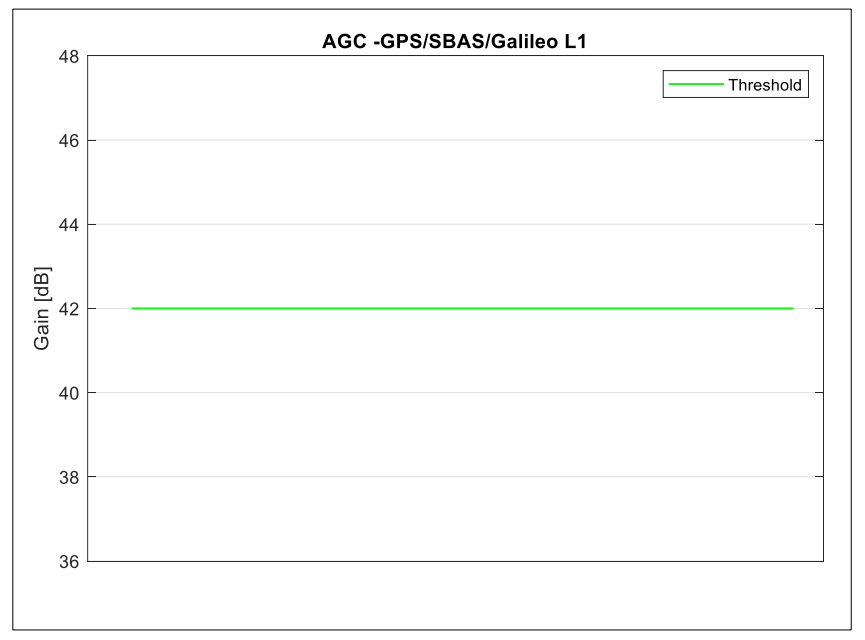

Figure 4: AGC level threshold 
The test mask for the PSD detection algorithm is an estimate of the power spectral density defined in absence of Electromagnetic Interference (EMI) in the frequency range of interest for the specific application and it is used as a reference to detect the presence of interference in the received GNSS SIS.

The RTCA DO-229D document specifies internally a mask. Such document contains the Minimum Operational Performance Standards (MOPS) for airborne navigation equipment using GPS augmented by Satellite-Based Augmentation System (SBAS) [17]. Therefore, such mask can be used in the interference detection process only if the receiver employed in the survey campaign is compliant with the standard. Moreover, it is important to take into account that the operational scenarios for an airborne GNSS receiver, in general, are different with respect to railway scenarios.

In case the receiver is not compliant with standard RTCA DO-229D, the mask has to be tailored to the specific receiver using the data gathered on field by the receiver itself. There are several sources of interference. For example, in the train there is a high number of equipment that could generate non-intentional harmful interference for the GNSS receiver and for the mask definition. In addition, the interference can also arise from the environment outside the train. For these reasons, it is better to have a static survey campaign. This allow characterizing the environment more easily in terms of interference.

Considering the data acquired in the operational conditions specified above, the next step is the definition of the mask, that is the estimation of the PSD, $\gamma\left(f_{n}\right)$, in the frequency range of interest.

At this aim, we recall that, when the receiver is not affected by interferences, the received signal contains the receiver noise plus the SISs whose amplitude, before CDMA demodulation, is far below the noise floor. The PSD of the received signal can be modelled statistically like a Gaussian distribution with expectation and variance given by the Power Spectral Density of the process.

Considering this, the estimation of the power spectral density on the interest frequency band and consequently the spectral mask estimation is carried out through the estimation of the power spectral density on the sub-intervals (or frequency bin) in which the entire frequency band has been partitioned initially.

Assuming the frequency band of interest has been partitioned into $N$ intervals and named $f_{n}$ the central frequency of the $n$-th frequency bin, the threshold value $\gamma\left(f_{n}\right)$ on each frequency $\operatorname{bin} f_{n}$, set in accordance to the desired false alarm probability $P_{f a}$, is equal to:

$$
\gamma\left(f_{n}\right)=P_{\underline{N}}\left(f_{n}\right)+\mathrm{K} * \sigma_{\underline{N}}\left(f_{n}\right) \quad \forall n=1, \ldots, N
$$

with

$$
\mathrm{K}=\operatorname{erfc}^{-1}\left(2 \sqrt{2} P_{f a}\right)
$$

$P_{\underline{N}}\left(f_{n}\right)$ and $\sigma_{\underline{N}}\left(f_{n}\right)$ are not known in advance and to compute them a Constant False Alarm Rate (CFAR) logic is applied. At this aim, we suppose that a PSD time series is available and defined as in the following:

$$
\left\{P S D_{i}\right\}_{i=1}^{I}
$$

where $P S D_{i}$ is the estimated power spectral density on the frequency band of interest at the time instant $i$ :

$$
P S D_{i}=\left\{\operatorname{PSD}_{i}\left(f_{1}\right), \operatorname{PSD}_{i}\left(f_{2}\right), \operatorname{PSD}_{i}\left(f_{3}\right), \ldots, P S D_{i}\left(f_{N}\right)\right\} .
$$

A partition of the discrete PSD time series $\left\{P S D_{i}\right\}_{i=1}^{I}$ in $M$ sub-intervals is then considered:

- Interval 1: $P S D\left(T_{1}\right)=\left\{P S D_{1}, P S D_{2}, P S D_{2}, \ldots, P S D_{k}\right\}$

- Interval 2: $P S D\left(T_{2}\right)=\left\{P S D_{k+1}, P S D_{k+2}, P S D_{k+3}, \ldots, P S D_{2 k}\right\}$

- $\quad \ldots$

- Interval M: $P S D\left(T_{M}\right)=\left\{P S D_{(M-1) k+1}, P S D_{(M-1) k+2}, P S D_{(M-1) k+3}, \ldots, P S D_{M k}\right\}$.

where $k$ is the number of elements in each interval. Finally, $P_{\underline{\underline{N}}}$ and $\sigma_{\underline{N}}$ are computed as follows:

$$
\begin{gathered}
P_{\underline{N}}=\text { Median }\left\{\overline{\operatorname{PSD}\left(T_{m}\right)}\right\} \quad \forall m=1, \ldots, M \\
\sigma_{\underline{N}}^{2}=\text { Median }\left\{\operatorname{Var}\left\{\operatorname{PSD}\left(T_{m}\right)\right\}\right\} \quad \forall m=1, \ldots, M
\end{gathered}
$$

where

$$
\begin{gathered}
\overline{P S D\left(T_{m}\right)}=\operatorname{mean}\left\{\operatorname{PSD}\left(T_{m}\right)\right\} \quad \forall m=1, \ldots, M \\
\operatorname{Var}\left\{\operatorname{PSD}\left(T_{m}\right)\right\}=\text { variance }\left\{P S D\left(T_{m}\right)\right\} \quad \forall m=1, \ldots, M
\end{gathered}
$$


However, the noise floor of each PSD fluctuates due to the presence of the AGC. Therefore, a pre-detection stage aimed at the AGC compensation has been introduced. This procedure equalizes the minima of the PSDs with respect to frequency, over time. In Figure 5 is shown a real example of the power spectral mask computed applying the described calibration algorithm. In this example the threshold has been computed using the PSD values depicted in Figure 3.

Considering the above, an interference is said to be present on a given time interval if exist at least one frequency bin of the considered frequency band in that time interval in which is verified the following condition $\operatorname{PSD}\left(f_{n}\right)>\gamma\left(f_{n}\right)$.

The decision threshold for both the detection techniques should be tailored to the specific measurement equipment using data gathered on the field. The measurement equipment for interference detection via AGC monitoring and PSD analysis is composed by a GNSS antenna and a GNSS receiver. In the train, other devices are also installed that should be considered as they are part of the final configuration of a specific train, like the on-board system, the signaling system, the engine, etc. For this reason, the calibration should be done for setting up the reference threshold considering that inside the train, due to the equipment installation, permanent noises / RFI signal sources can be present that are independent from the environment outside the train.

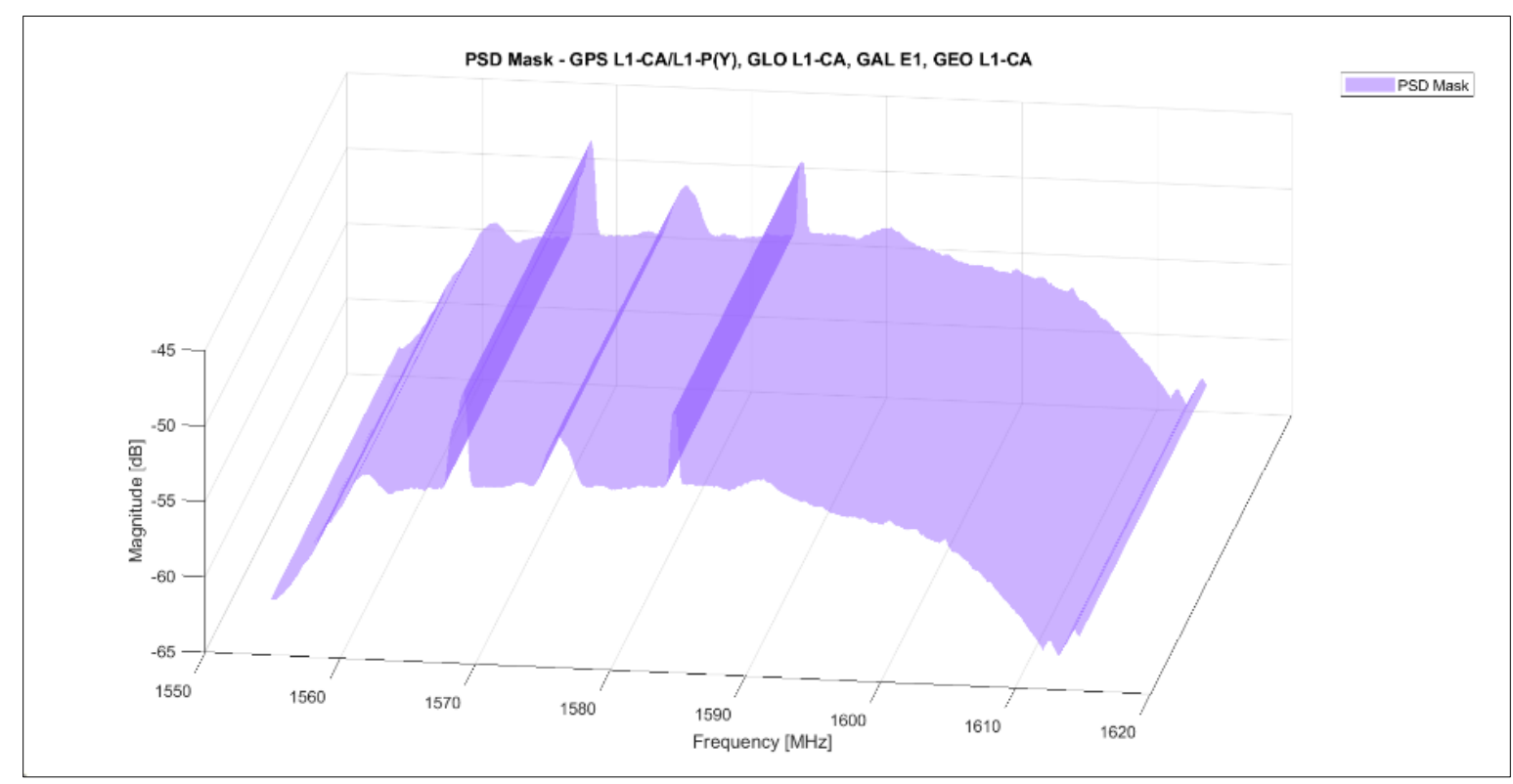

Figure 5: PSD mask

\section{INTERFERENCE DETECTION ALGORITHM}

Since a unified approach applicable to all types of interference is not available, but only specific interference characteristics can be addressed by a single detection method, the combination of different metrics can be necessary. This approach can reduce erroneous interference detection caused by other interfering signals such as multipath allowing to correctly identify RFI events and to decrease the false-alarm detection probability. The proposed algorithm has been designed considering two different techniques in order to integrate different decision metrics and create a unified decision for the detection of the presence of interference in a certain area of the railway track. In particular, the interference detection algorithm is based on the output combination of the AGC and the PSD techniques. The combination of the outputs produced by these two metrics allows to reduce the probability of false alarm to declare an EMI event with respect to the same false alarm probability characterizing each one of these techniques considered individually. Both the AGC technique and the PSD analysis provide a binary output: 1 (true) or 0 (false). Specifically, the 1 and 0 values define whether the test statistic value for each metric is above the threshold or not. In order to decide if an interference is present in a specific epoch of interest and declare consequently that an EMI event is occurred, an AND logic function has been defined on the results from the two different metrics as described in the Table 1. In summary, in a specific time epoch, an EMI event is occurred if and only if the test statistic value for both metrics are above the threshold. 
Table 1: Decision rule

\begin{tabular}{|c|c|c|}
\hline AGC & PSD & STATUS \\
\hline $\mathbf{1}$ & $\mathbf{1}$ & EMI detected \\
\hline $\mathbf{1}$ & $\mathbf{0}$ & No EMI \\
\hline $\mathbf{0}$ & $\mathbf{1}$ & No EMI \\
\hline $\mathbf{0}$ & $\mathbf{0}$ & No EMI \\
\hline
\end{tabular}

In Figure 6 is depicted the conceptual scheme of the entire process of EMI detection. In the first phase of the process, the data are recorded by the measurement equipment on-board the train while it is performing the runs in order to gather the raw data necessary to cover the dynamics of the GNSS signal along the line. The collected data are then processed offline in order to adapt them to the input format of the Detection methods. In the second phase, the EMI anomaly Detection methods block passes the collected measurements to the AGC and PSD monitoring methods. Each detection method is independent and individually provide a series of detection flags in the time domain based on the input data. Then, in the EMI detection algorithm block, the results of the two detection techniques are combined, as described above, to obtain the EMI detection results. Finally, the block Location mapping reports the results of the EMI Detection block from the time domain into space domain in order to locate the point on the railway track where the interference event is occurred.

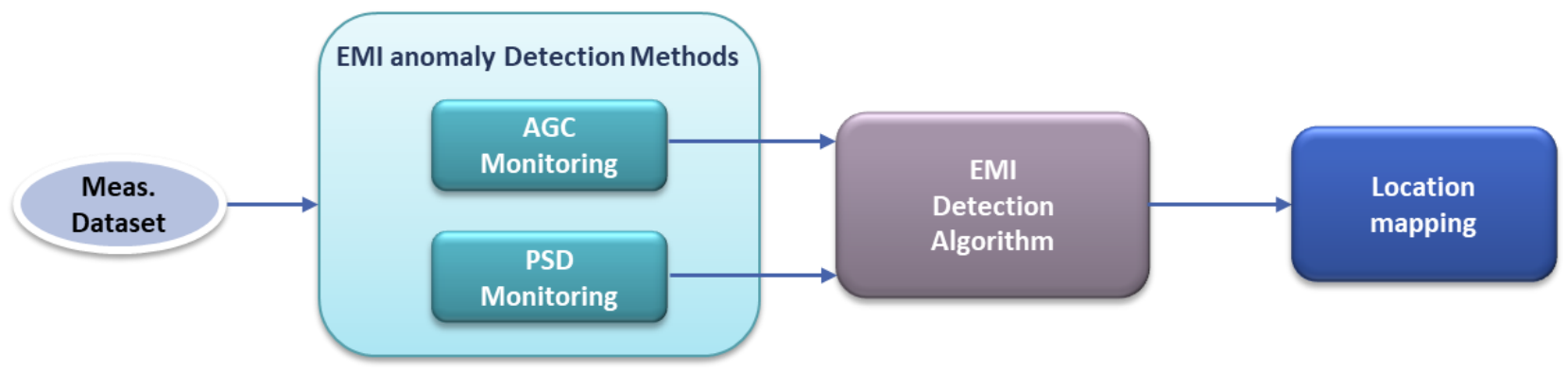

Figure 6: Conceptual scheme of the interference detection algorithm

\section{MEASUREMENTS CHAIN SET-UP}

In order to assess the performance of the proposed EMI detection algorithm, the data gathered during the measurement campaign carried out in the context of the ERSAT-GGC project and the processing tool designed and developed in the same project have been used. Particularly, the measurement campaign has been carried out in Sardinia using the testbed provided by Rete Ferroviaria Italiana (the Italian railways infrastructure manager). The trial test site is located on the Cagliari - San Gavino line (Figure 7). The importance of this trial site is in the fact that here it has been started the tests on the new architecture that integrates the concept of Virtual balise based on GNSS technology with the ERTMS/ETCS. Other general information of the trial site is listed in the following:

- Total length of the line: $50 \mathrm{~km}$ (Cagliari- San Gavino)

- Double Track line

- Low-traffic line that allowed this type of activity to be carried out compatibly with commercial service activities

- Maximum speed $150 \mathrm{~km} / \mathrm{h}$

- Augmentation network with two reference stations

- 3 Tetra Antenna in Cagliari, Decimomannu and Samassi

- $\quad$ Vehicle ALn668 - 3136 

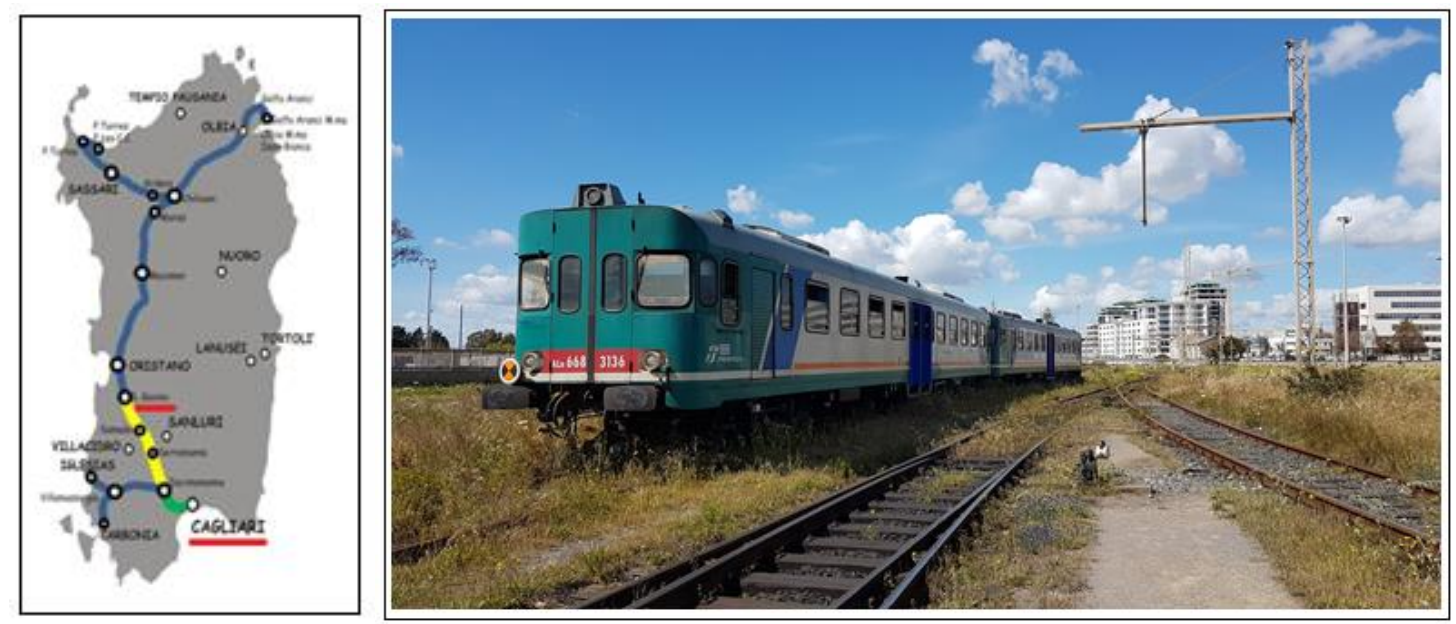

Figure 7: Sardinia testbed line and ALn668 - 3136 train

The assessment activity of the algorithm performances used only a subset of the facility make available in the trial site. Specifically, only the facility on-board the train has been used. The equipment was placed in a specific cabinet located in the luggage area just behind the driving cab. The same area can host a desk for the measurement instrumentation. Figure 8 shows the schematic diagram of measurement chain.

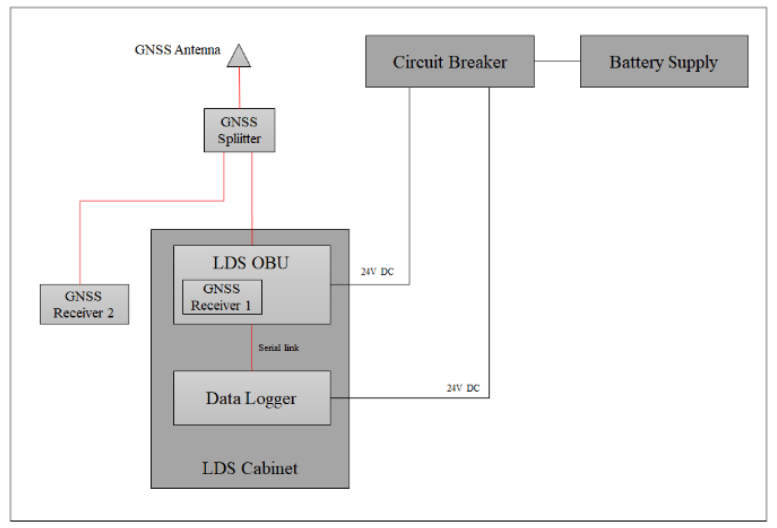

Figure 8: Experimental set-up

The GNSS SIS is captured by the Antcom's G5 antenna installed on the train roof as shown in Figure 9. It is an active L1/L2/L5 GPS + E1/E5/E6 GALILEO antenna.

The GNSS splitter (ZB4PD1-2000-S+) directly connected to the antenna, supplies two different GNSS receiver as depicted in Figure 8. Specifically, the first GNSS Receiver is a Septentrio AsteRx4 OEM receiver. It is a multi-frequency and multi-constellation (GPS, GLONASS, Galileo, BeiDou, IRNSS and QZSS) dual antenna. As shown in the Figure 2, this equipment belongs to the location determination system (LDS) of the on-board unit (OBU).

The second used GNSS receiver is a Javad Delta-3N receiver. It is a geodetic multi-frequency and multi-constellation (GPS, GLONASS, Galileo, BeiDou, IRNSS and QZSS) receiver. This equipment has been used in the measurement campaign in addition to that in the LDS OBU, in order to evaluate the algorithms performance on data gathered by different receivers. It is important to consider that the different receivers available on the market do not provide exactly the same information. This means that in general some adaptation/pre-processing of data is necessary to apply the techniques described above. For example, in case of the spectral monitoring technique, it takes as input the power spectral density. The Javad Delta-3N receiver provides in output this type of information that therefore is put in input directly to the spectral monitoring technique. Instead, the Septentrio AsteRx4 OEM receiver make available the I/Q samples as output to the ADC block (Figure 1). Therefore, the power spectral density has to be computed. Starting from this type of information, the Welch's method has been used estimating the power spectral density of the signal in the 
ERSAT-GGC project. This method is based on the periodogram method which in turn use the sequences of shorter and windowed fast Fourier transforms.

The Automatic Gain Control monitoring technique takes as input the gain values in output to the AGC blocks. Currently, there is no standardization among GNSS receivers for AGC reporting units or the measurement itself. Most COTS receivers offer such a metric, but it is likely that each needs to be interpreted individually [11]. In this case, both the JAVAD and Septentrio receivers provide in output the AGC level values, therefore no adaptation is necessary.

In summary, the registered measurements during the campaign have been:

- The baseband I/Q samples and the AGC level values for the SEPTENTRIO ASTERX4 OEM receiver

- The PSD values and the AGC level values for the Javad Delta-3N.

The entire measurement campaign has been designed to gather two types of measurements

- Static measurements

- Dynamic measurements.

The static measurements are relative to the train in standstill condition, positioned close to the railway station in open sky environment. This data has been used for the calibration of the PSD and AGC monitoring techniques. Overall, eight hours of data have been collected. The static measurements were collected with all the train systems and engine switched on, in order to capture any degradation of AGC and PSD due to the nominal electronics of the train.

The dynamic measurements, instead, were collected while the train was operated between the Cagliari and San Gavino stations. These data have been used into the performance evaluation activity. In this case, about eighteen hours of data have been collected on thirty-four runs: seventeen runs from Cagliari to San Gavino and seventeen from San Gavino to Cagliari. Overall, the measurement campaign lasted seven days.

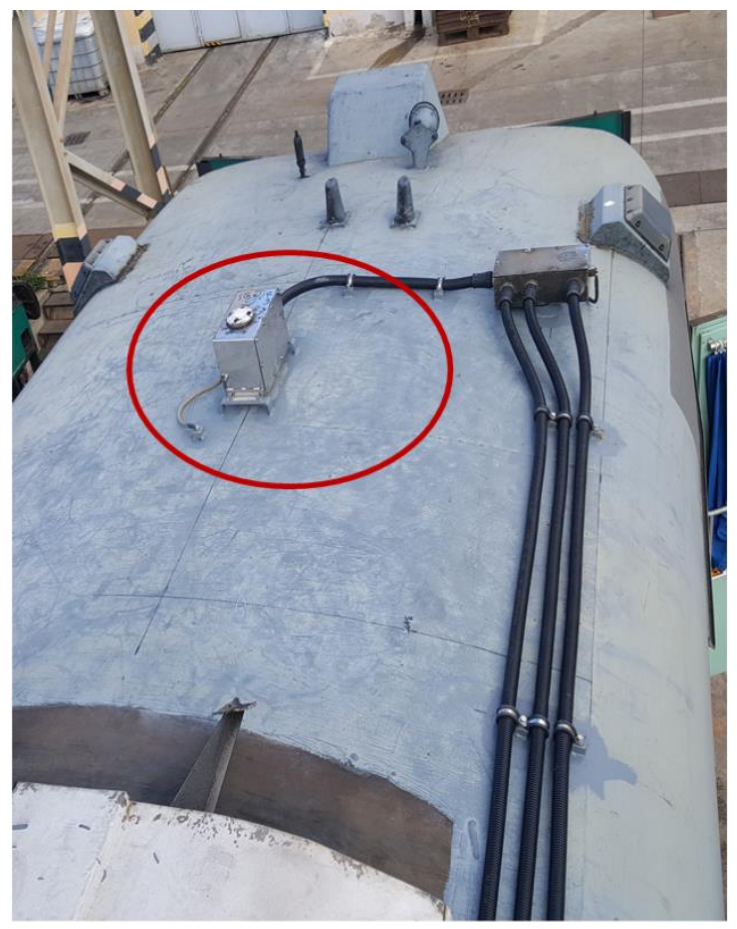

a. Antcom's G5 antenna

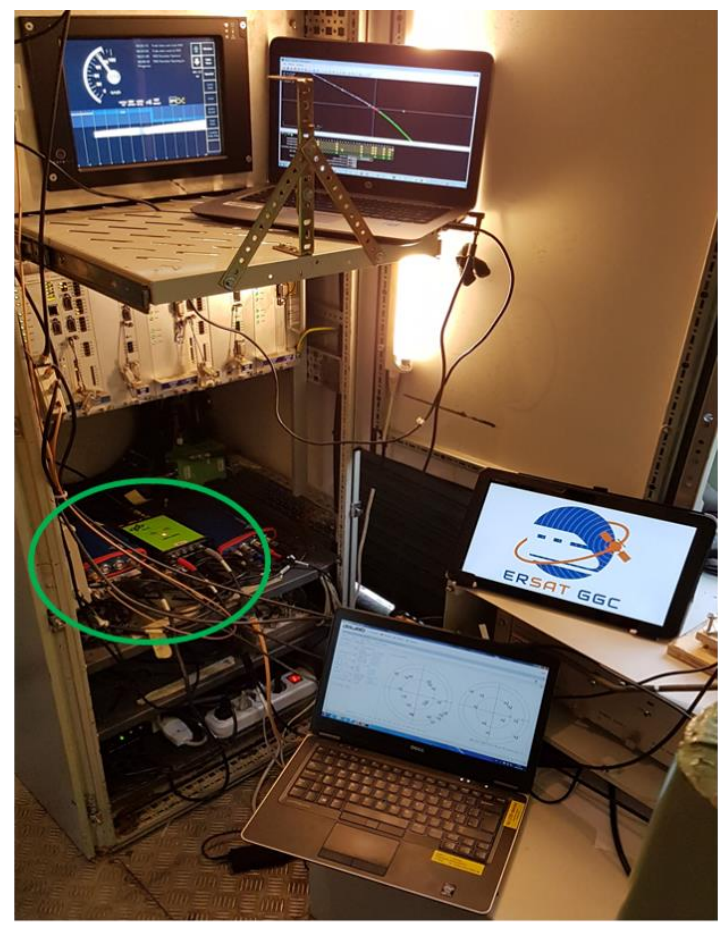

b. GNSS receivers

Figure 9: Measurement sub-system installation in the train

Two GNSS receivers have been used during the measurement campaign in Sardinia in order to allow a comparative analysis of the detection results; this comparative analysis has been done for the verification of the interference detection process. One receiver only 
is required for the application of the interference detection process. Both receivers have been configured to record the following data in the proprietary format, for multiple constellations and multiple frequencies:

- Time tag in TOW (time of week expressed in ms) and WNc (week number counter)

- Carrier to noise ratio in $\mathrm{dB}-\mathrm{Hz}$

- I and Q sample or spectrum data

- $\quad$ AGC data.

\section{RESULTS}

In order to assess the performance of the proposed interference detection algorithm, data acquired during the measurement campaign carried out in the context of the ERSAT-GGC project have been used. Figure 10 and Figure 11 show the behavior of the PSD and AGC level acquired with the Septentrio AsteRx4 receiver during the same interference event. The blue line in the AGC plot represents the AGC level variation over time, while the green line is the decision threshold. The blue trend in the PSD plot represents the PSD variation over time, while the purple surface is the decision mask.

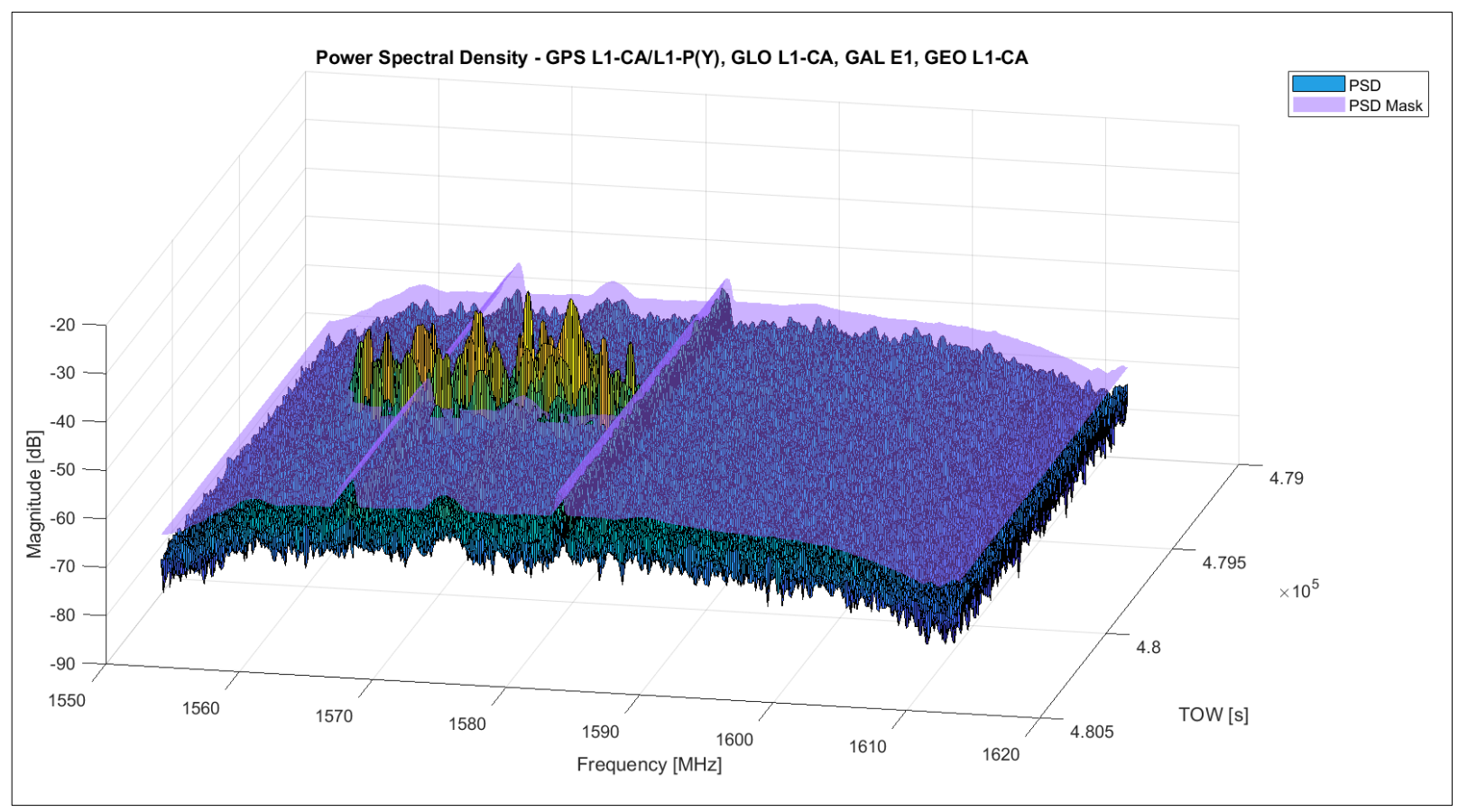

Figure 10: Example of interference anomaly detection with PSD monitoring 


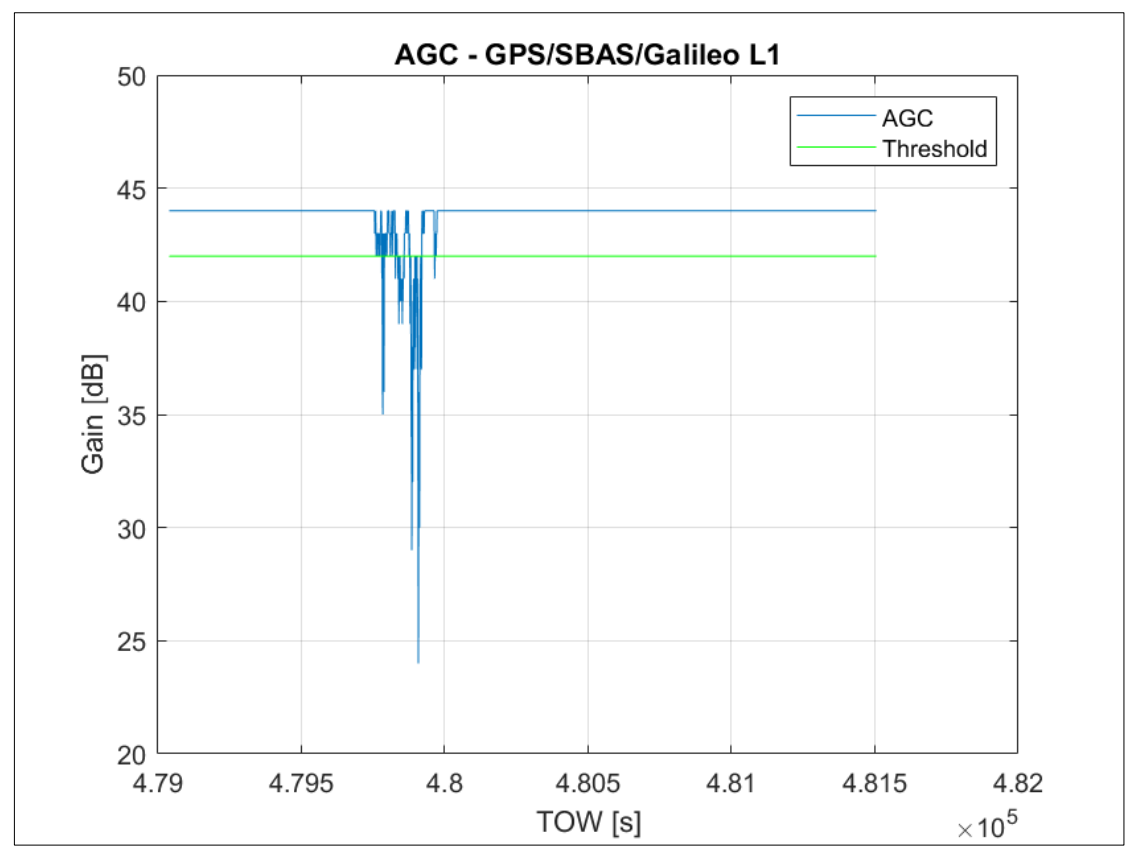

Figure 11: Example of interference anomaly detection with AGC level monitoring

A comparative analysis among interference detection results obtained with the two receivers in the space domain is shown in Figure 12, Figure 13 and Figure 14. In particular, the pictures show how the same interference occurrences have been detected on the line using both datasets acquired with Septentrio and Javad receivers. The comparative analysis, here presented as verification evidence, shows that the interference detection process produces similar results when using different receivers. Therefore, the interference detection results are receiver independent.

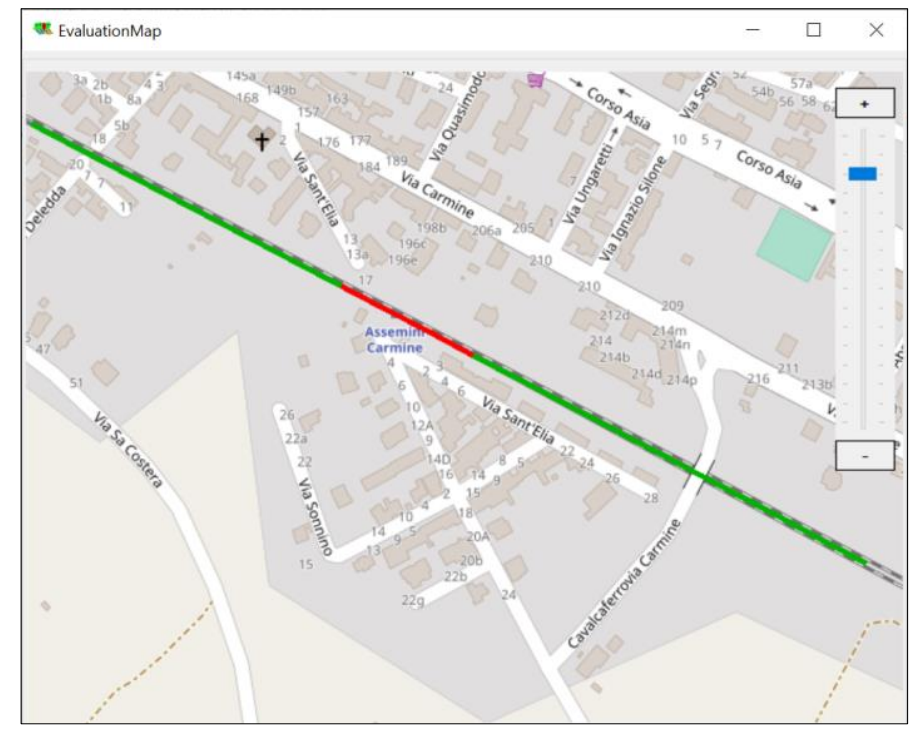

Septentrio AsteRx4 OEM

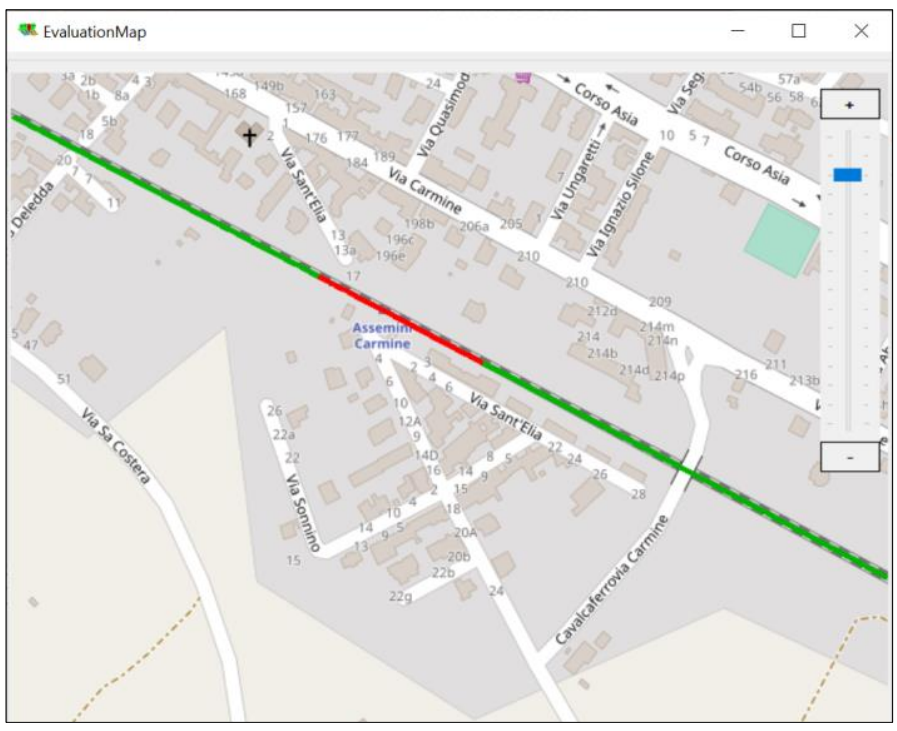

Javad Delta-3N

Figure 12: Comparison of the interference detection results at Assemini station 


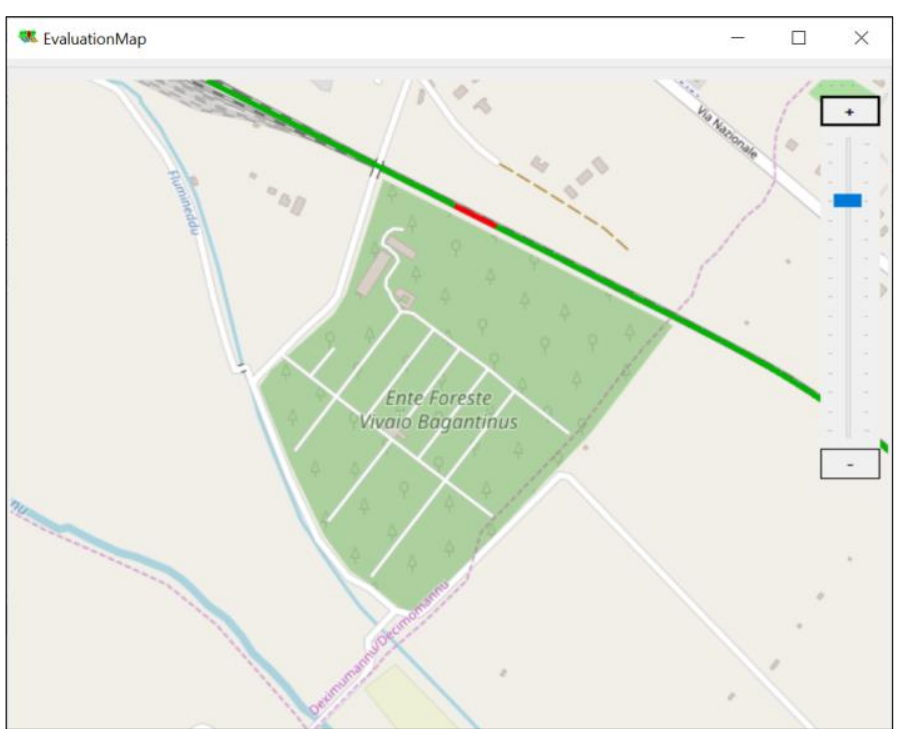

Septentrio AsteRx4 OEM

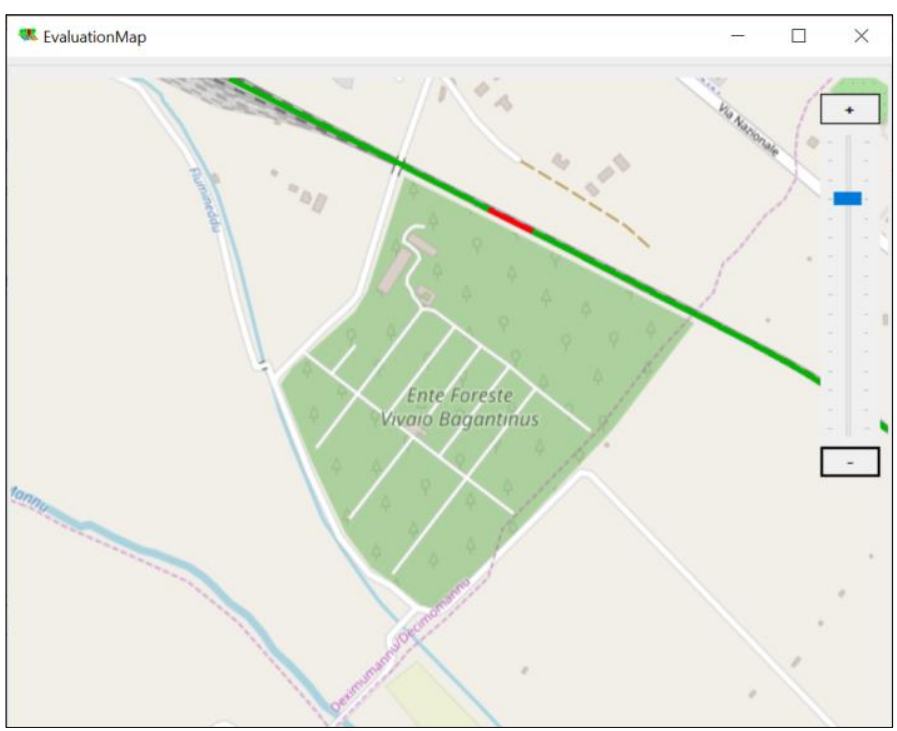

Javad Delta-3N

Figure 13: Comparison of the interference detection results near Decimomannu station

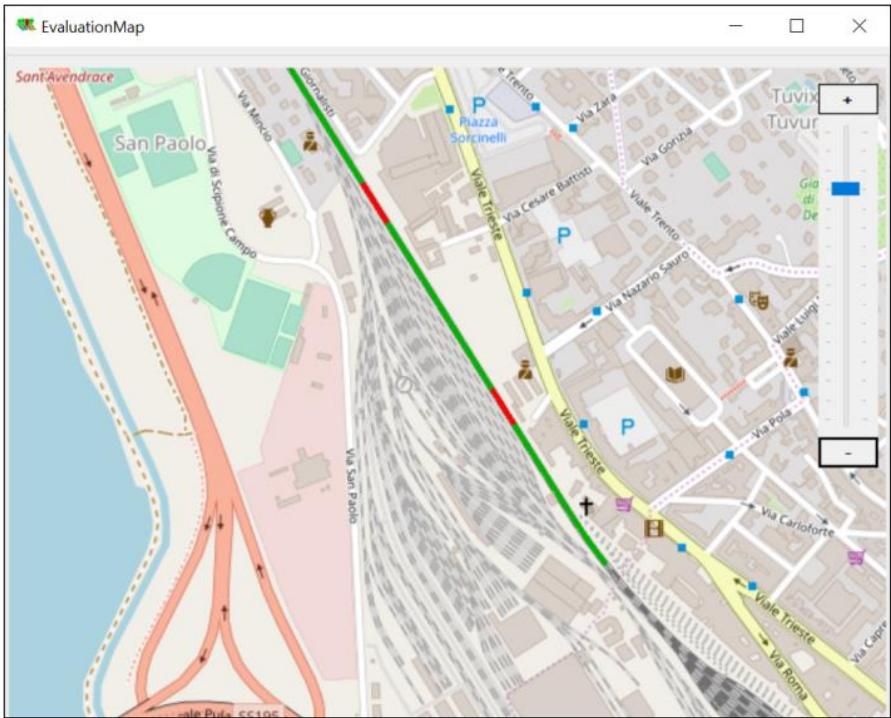

Septentrio AsteRx4 OEM

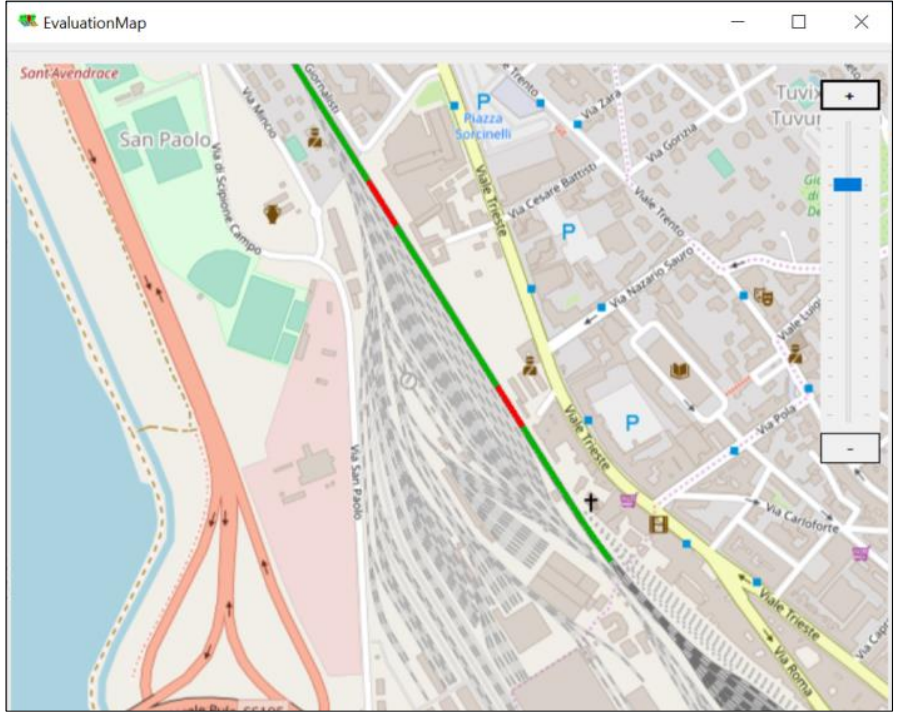

Javad Delta-3N

Figure 14: Comparison of the interference detection results at Cagliari station

\section{CONCLUSION}

This work has presented the capability of detection of interference in the railway environment. Since the detection techniques are based on measurements or information available in most COTS receivers, it can be applied to different types of receivers. The methodology presented ensures a robust interference detection by the combination of two different monitoring techniques. They are well interfaced together to produce combined results both in time and space domains along the railway track lines. It is here stressed 
the importance of a dedicated calibration process to properly determine the detection thresholds for specific railway installations. We showed the applicability of this detection techniques to an overall classification process of the railway tracks with respect to the expected presence of interference by the accumulated detection data. The technique and classification information will be important for the safe use of GNSS in future railway applications.

\section{ACKNOWLEDGMENTS}

This work has been funded by the European GSA H2020 ERSAT-GGC project. The authors would like to thank all the partners of ERSAT-GGC project. In particular, Trenitalia and Rete Ferroviaria Italiana (RFI) that made available the train and the line for the measurements during the project and Hitachi Rail STS for the installation of the antenna and equipment on the train.

\section{REFERENCES}

1. I. Hughes and T. Hase, Measurements and their Uncertainties A Practical Guide to Modern Error Analysis, Oxford University Press, Oxford, 2010.

2. ESA 3InSat, [Online]. Available: https://business.esa.int/projects/3insat.

3. ESA SBS, [Online]. Available: https://business.esa.int/projects/sbsrails.

4. ERSAT-EAV European GNSS Agency (GSA), [Online]. Available: http://www.ersat-eav.eu/.

5. RHINOS project, Railway high integrity navigation overlay system, [Online]. Available: https://www.gsa.europa.eu/railwayhigh-integrity-navigation-overlay-system

6. Massimiliano Ciaffi, Juliette Marais, Giusy Emmanuele, Omar Garcia Crespillo, Andrea Coluccia, Alessia Vennarini, Salvatore Sabina, Syed Ali Kazim, Daniel Gerbeth, Maria Caamano, Alessandro Neri, Elena Razzano, Fabio Senesi, "Classification of Railway tracks for applying enhanced ERTMS/ETCS Solutions Based on GNSS positioning technologies", WCRR 2019 - World Congress on Railway Research, At Tokyo, Japan

7. M. Caamano, O. García Crespillo, D. Gerbeth and A. Grosch, "Detection of GNSS Multipath with Time-Differenced CodeMinus-Carrier for Land-based Applications," in 2020 European Navigation Conference (ENC), Dresden, Germany, 2020

8. Marais, Juliette, Hmida, Rihab, Flancquart, Amaury, Sabina, Salvatore, Ciaffi, Massimiliano, "Video-based Classification of Railway Track Areas for GNSS-based Virtual Balise Solutions in the ERSAT GGC Project," Proceedings of the 2018 International Technical Meeting of The Institute of Navigation, Reston, Virginia, January 2018, pp. 196-205.

9. Garcia Crespillo, Omar and Konovaltsev, Andriy and Marais, Juliette and Sabina, Salvatore and Vennarini, Alessia and Coluccia, Andrea and Neri, Alessandro and Aguila, Antonio and Razzano, Elena and Ranauro, Francesco and Ciaffi, Massimiliano (2018) Local GNSS Threat Detection Methods for Virtual Balise Placement in Railway Applications. 2018 16th International Conference on Intelligent Transportation Systems Telecommunications (ITST), 15-17 Oct 2018, Lisbon, Portugal

10. D. Akos, "Who's Afraid of the Spoofer? GPS/GNSS Spoofing Detection via Automatic Gain Control (AGC)," Journal of Navigation, vol. 59, no. 4, pp. 281-290, 2012.

11. H. Borowski, O. Isoz, F. M. Eklöf, S. Lo and D. Akos., "Detecting False Signals with Automatic Gain Control," GPS World, 29 February 2016. [Online]. Available: http://gpsworld.com/detecting-false-signals-automatic-gain-control-1280.

12. F. Dovis, GNSS Interference Threats and Countermeasures, Artech House, 2015.

13. F. Bastide, D. Akos, C. Macabiau and B. Roturier, "Automatic Gain Control (AGC) as an Interference Assessment Tool," in ION GPS, Portland, 2003.

14. M. Z. H. Bhuiyan, H. Kuusniemi, S. Söderholm and E. Airos, "The Impact of Interference on GNSS Receiver Observables - A Running Digital Sum Based Simple Jammer Detector,” 2014.

15. E. G. Manfredini, D. M. Akos, Y.-H. Chen, S. Lo, T. Walter and P. Enge, "Effective GPS Spoofing Detection Utilizing Metrics from Commercial Receivers," in Proceedings of the 2018 International Technical Meeting of The Institute of Navigation, Reston, VA, 2018.

16. M. Pini, B. Motella and L. L. Presti, "Comparison Between AGC Control and Statistical Based Methods for Low Power Interference Detection," in Proceedings of the 29th International Technical Meeting of the Satellite Division of the Institute of Navigation (ION GNSS+), Portland, OR, 2016

17. RTCA DO-229D, "Minimum Operational Performance Standards for Global Positioning System/Wide Area Augmentation System Airborne Equipment", 2006. 